\title{
Aerodynamics of a Successful Perching Maneuver
}

\author{
Tyler P. Krone ${ }^{1}$, Yeon Sik Baik ${ }^{2}$, Jonathan M. Rausch ${ }^{3}$, Luis P. Bernal ${ }^{4}$ \\ University of Michigan, Ann Arbor, MI
}

\begin{abstract}
An experimental study of a perching maneuver performed by a flat plate at $\mathrm{Re}-\mathbf{3 5 , 0 0 0}$ is presented in this paper. The autonomous perching maneuver of a UAV that consists of a pitch-up motion at moderate pitching rate is used in the study. Experiments were conducted at the University of Michigan's low-turbulence water channel facility in two different phases. The first phase was flow visualization by dye injection. The second phase was flow field calculation using 2D phase-averaged particle image velocimetry (PIV). The behavior of the flow field was studied at $\mathbf{5 0 \%}$ and $\mathbf{7 5 \%}$ span-wise locations during each phase. The formation of a separation bubble followed by the shedding of the trailing edge vortex (TEV) is observed. Vorticity is introduced at the leading edge of the flat plate, and can be traced downstream and observed in the recirculation zone.
\end{abstract}

\section{Nomenclature}

$\alpha \quad=$ Angle of attack $\left[{ }^{\circ}\right]$

$c \quad=$ Chord $[m]$

$k=\dot{\theta} c / 2 U$, Reduced frequency [1]

Re $\quad=U c / v$, Reynolds number [1]

$t \quad=$ Time $[s]$

$t_{c} \quad=U t / c$, Convective time [1]

$U \quad=$ Flow speed $[\mathrm{m} / \mathrm{s}]$

$v \quad=$ Kinematic viscosity $\left[\mathrm{m}^{2} / \mathrm{s}\right]$

$\theta \quad=$ Pitch angle $\left[{ }^{\circ}\right]$

$\dot{\theta}=$ Pitch rate $[\%]$

$\theta_{\rho} \quad=\quad \dot{\theta} c / U$, Non-dimensional pitch rate [1]

\section{Introduction}

The study of biologically-inspired transient aerodynamic maneuvers has been a subject of interest for aerodynamicists seeking to both understand natural flight and create bio-inspired Unmanned Aerial Vehicles (UAV). Perching is a particularly intriguing motion performed by birds that allows them to land at precise locations without the need to decelerate on the ground. Cory and Tedrake ${ }^{1}$ studied perching from a controls perspective, and were able to develop an autonomous controller capable of perching a fixed-wing glider in an indoor motion capture environment ${ }^{1}$. In their study the Reynolds number at the beginning of the glider motion was 43,000 , while it was 5,000 at flight termination ${ }^{1}$. The present study characterizes the flow field properties of Cory and Tedrake's ${ }^{1}$ specific transient maneuver. Flow visualization by dye injection and PIV analysis of the flow field downstream of the flat plate during the perching maneuver are used to quantify the flow field. The flow visualization results presented in this paper demonstrate that perching maneuvers create a separated flow field with a large separation bubble. McCroskey ${ }^{2}$ showed the presence and passage of a leading edge vortex (LEV) can increase the lift coefficient beyond the linear region, which may help explain the success of the perching maneuver.

\footnotetext{
${ }^{1}$ Graduate Student, University of Michigan, Department of Aerospace Engineering, tkrone@umich.edu

${ }^{2}$ Graduate Research Assistant, University of Michigan, Department of Aerospace Engineering, yeonb@umich.edu

3 Graduate Research Assistant, University of Michigan, Department of Aerospace Engineering, rauschjm@umich.edu

${ }^{4}$ Associate Professor, University of Michigan, Department of Aerospace Engineering, 1pb@umich.edu
} 
Investigating the perching motion improves the understanding of unsteady transient maneuvers. $\mathrm{Ol}^{3}$ investigated the high-frequency, high-amplitude pitching motion of various wings in a water tunnel, while Elderege, Wang, and $\mathrm{Ol}^{4}$ studied this problem computationally. Elderege, Wang, and $\mathrm{Ol}^{4}$ found evidence the LEV was crucial in lift generation, providing insight as to why this maneuver is successful.

Wickenheiser, Garcia, and Waszak ${ }^{5}$ investigated the longitudinal dynamics and controls of a morphing, perching aircraft. Hoburg and Tedrake ${ }^{6}$ expanded on the longitudinal control work done by Cory and Tedrake ${ }^{1}$. The major difference between the experimental testing done by $\mathrm{Ol}^{3}$ (water tunnel) and that of Cory and Tedrake ${ }^{1}$ and Hoburg and Tedrake ${ }^{6}$ (flight data) is that the flow speed and consequently Re changed over the course of the motion in the latter two experiments.

The inability to change $R e$ throughout the perching motion is a major limitation of running experiments in the water channel, but recent results $\mathrm{Ol} \mathrm{et} \mathrm{al}^{9}$ show that at the Reynolds number and deceleration rates considered here the lift and drag coefficients are only weakly dependent on Reynolds number except at very low speeds.

\section{Experimental Setup}

Experiments were conducted in the low-turbulence water channel in the Francois-Xavier Bagnoud (FXB) building at the University of Michigan (UM). The low-turbulence water channel has a test cross-section of $61 \mathrm{~cm}$ wide by $61 \mathrm{~cm}$ high, with a turbulence intensity of approximately $1 \%{ }^{7}$ Fig. 1 shows the water channel facilities at UM.

The test model is a $2.3 \%$ thick flat stainless-steel square plate with rounded leading and trailing edges mounted normal to the water surface. The model chord and semi-span are $152 \mathrm{~mm}$. The model is mounted on an end wall to simulate a symmetry plane and mitigate water free-surface effects. The end wall is $635 \mathrm{~mm} \times 405 \mathrm{~mm}$ and contains a $295 \mathrm{~mm}$ diameter circular center section which rotates with the model as shown in Fig. 1. The effective aspect ratio of test wing is 2 . This aspect ratio corresponds well to the 2.66 aspect ratio of one of the glider's wing used by Cory and Tedrake ${ }^{1}$. The flat plate is polished to minimize diffuse reflection from the laser beam and glare near the surface. ${ }^{7}$ This setup allowed for some model flexibility, but it was shown by Baik et al. that this was minimal. ${ }^{7}$

The model and circular portion of the end wall rotate about the quarter-chord point of the flat plate. The pitch motion was produced by a rotary stage (Velmex B4872TS Rotary Table) and the associated computer control system (Velmex VXM-1-1 motor control). ${ }^{7}$ As discussed below, the motion kinematics are scaled to simulate the perching motion of Cory and Tedrake's ${ }^{1}$ experiment.

The flow visualization system used in the UM water channel consists of seven uniformly distributed dye streams introduced several chords upstream of the leading edge of the model. Two syringe pumps were used to match the dye injection speed with the water channel speed to help minimize dye stream disturbances. The dye streams were held fixed and the model was pitched accordingly.
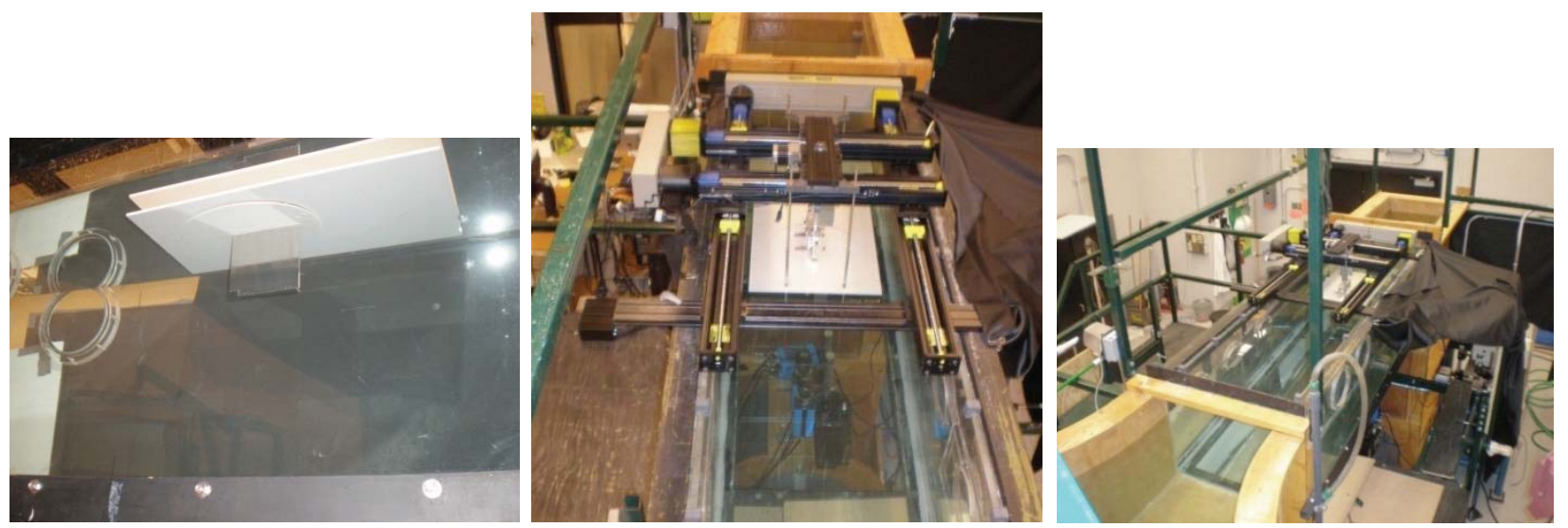

Figure 1. Water channel at the University of Michigan. Flat plate mounted with endplate, freestream flows left to right (LEFT), VELMEX rotary table with mounted flat plate, freestream flows top to bottom (MIDDLE), water channel test section, PIV system located to the right of the channel (RIGHT). 
The PIV system at the University of Michigan included a double-pulsed Nd-YAG laser (Spectra Physics PIV 300), light sheet formation optics, two dual frame digital cameras (Cooke Corp. PCO-4000), computer image acquisition system, and control electronics. For PIV image acquisition, the water channel was seeded with $5 \mu \mathrm{m}$ diameter Titanium Dioxide particles. A small amount of a dispersant (DARVAN C-N, Vanderbilt) was used to produce a uniform distribution of particles and to help maintain the particles in suspension for long periods of time of the order of several days. The two cameras were installed underneath the water channel test section and were equipped with Nikon 105-mm focal length Micro-Nikkor lenses to produce matching magnifications of approximately 18 pixels $/ \mathrm{mm}$. Each camera has a sensor size of 4008 × 2672 pixels and the cameras were setup with a 469 pixel overlap in order to capture the large separated flow region behind the flat plate. The resulting field of view is approximately $4875 \times 4008$ pixels, which at the present magnification corresponds to $271 \times 223 \mathrm{~mm}$. The time between exposures was calculated to produce a nominal particle displacement of 8 pixels at the free stream velocity corresponding to $R e=35,000{ }^{7}$

For the present experiments the pitching motion of Cory and Tedrake's ${ }^{1}$ experiment scaled for the present water tests as discussed below was divided into 26 individual phases spaced 1 second apart. A total of 115 PIV images of the flow field were recorded at each phase and used to calculate phase-averaged mean flow fields. See Baik et al. ${ }^{7}$ for PIV system specifics and in-house MATLAB-based PIV analysis software details.

\section{Results}

\section{A. Pitch-Hold Kinematics}

Cory and Tedrake ${ }^{1}$ provided sample data of a successful perching maneuver which is illustrated in Fig. 2. The maneuver consists of a rapid pitch-up rotation about the center of gravity and simultaneous deceleration due to the increased drag. Relevant non-dimensional parameters are the Reynolds number $(\mathrm{Uc} / \mathrm{v})$, convective time $(\mathrm{U} \mathrm{t} / \mathrm{c})$, the pitch angle $(\theta)$ and the non-dimensional pitch rate $(\dot{\theta} \mathrm{c} / \mathrm{U})$. Cory and Tedrake's ${ }^{1}$ data in non-dimensional form are plotted in Fig. 3. Also plotted in Fig. 3 are the same non-dimensional parameters for the present experiments.
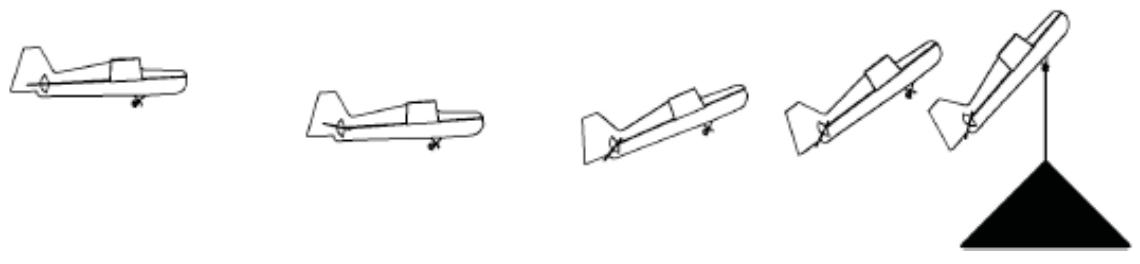

Figure 2. Visualization of Cory and Tedrake's ${ }^{1}$ glider performing a successful perching maneuver. The entire trajectory took $\sim 0.9$ seconds.

An important difference between the actual perching maneuver and the present experiment is that deceleration of the glider results in reduction of flow speed and consequently Reynolds number from approximately 43,000 to 5,000 . Recent experimental results by $\mathrm{Ol}$ et $a l^{9}$ show that deceleration for moderate reduced frequency $(\mathrm{k}=\dot{\theta} \mathrm{c} / 2 \mathrm{U})$ has small effect on the lift coefficient. Motivated by Ol et al $l^{9}$ results, Cory and Tedrake's ${ }^{1}$ maneuver was simulated in the water channel using constant $R e=35,000$ which corresponds to the $R e$ for maximum non-dimensional pitch rate. In addition, the same non-dimensional pitch rate as in Cory and Tedrake's ${ }^{1}$ maneuver was used in the present experiment. The pitch angle was computed from the non-dimensional pitch rate and it is shown as a dotted line in Fig. 3. Because non-dimensional pitch rate was matched between the experiment and the actual perching maneuver the two lines in Fig. 3 (right) overlap. Note that the maximum pitch angle for the present experiments is lower by 1 degree compared to the maximum pitch angle for Cory and Tedrake's ${ }^{1}$ maneuver. 

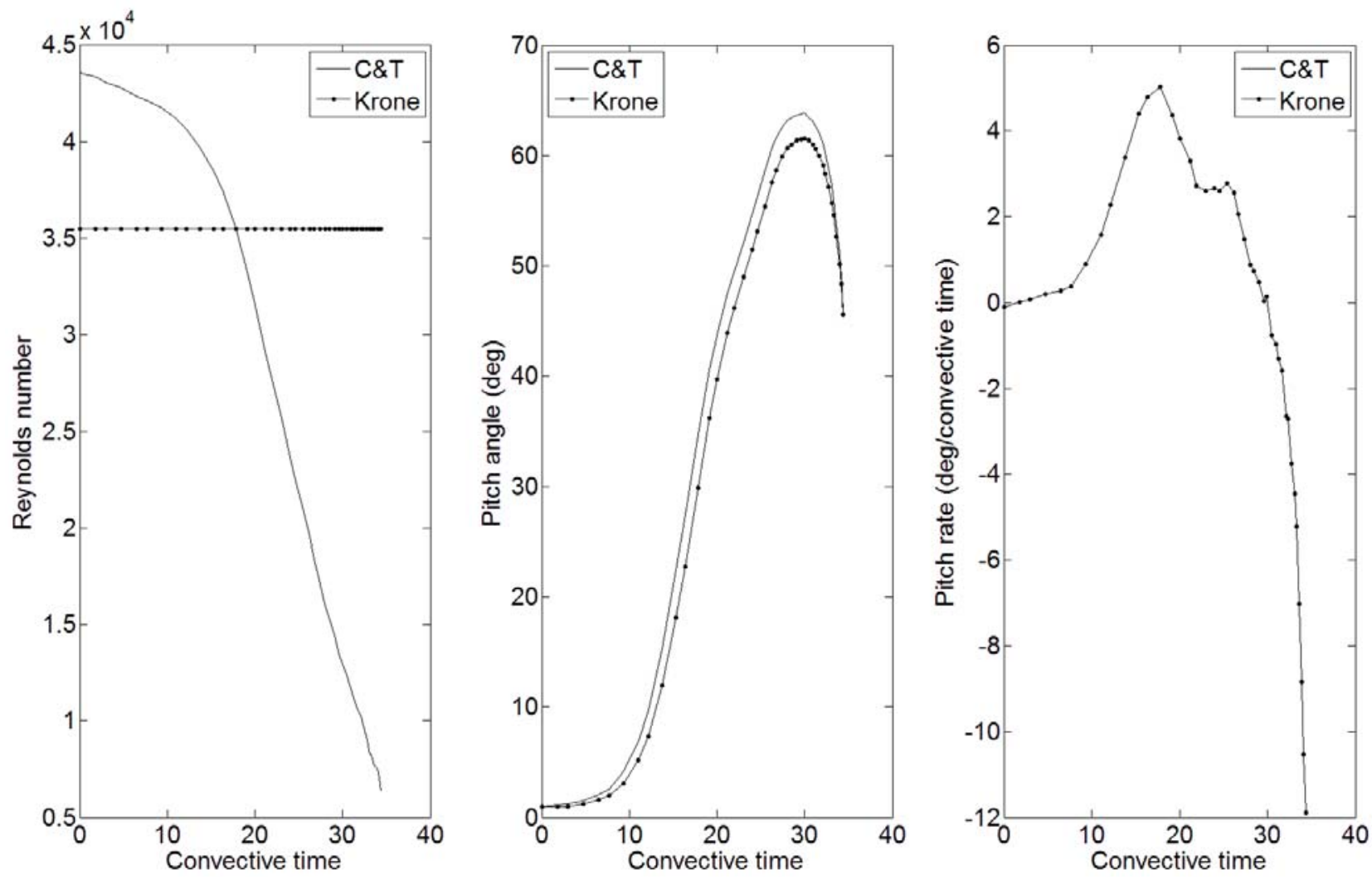

Figure 3. Glider motion recorded by Cory and Tedrake ${ }^{1}$ for a glider performing a perching maneuver in terms of the relevant non-dimensional parameters. Left: Reynolds number (glider speed) as a function of convective time. Center: Pitch as a function of convetive time. Right: Non-dimensional pitch rate as function of convective time. Shown in the same plots is the corresponding parameters for the water channel experiments reported here.

As noted earlier, the results of $\mathrm{Ol}$ et al. ${ }^{9}$ suggest that the scaling approach is justified only if the reduced frequency, $k$, is small. To validate this assumption Fig. 4 plots the pitch angle and reduced frequency as a function of time for the present experiments. The reduced frequency is below 0.04 for times less than 25 seconds, which corresponds to a convective time of 31.5 , and the present scaling approach should be valid. For greater convective time the flow velocity in Cory and Tedrake's ${ }^{1}$ experiment is very low and the present scaling is not expected to be valid. 


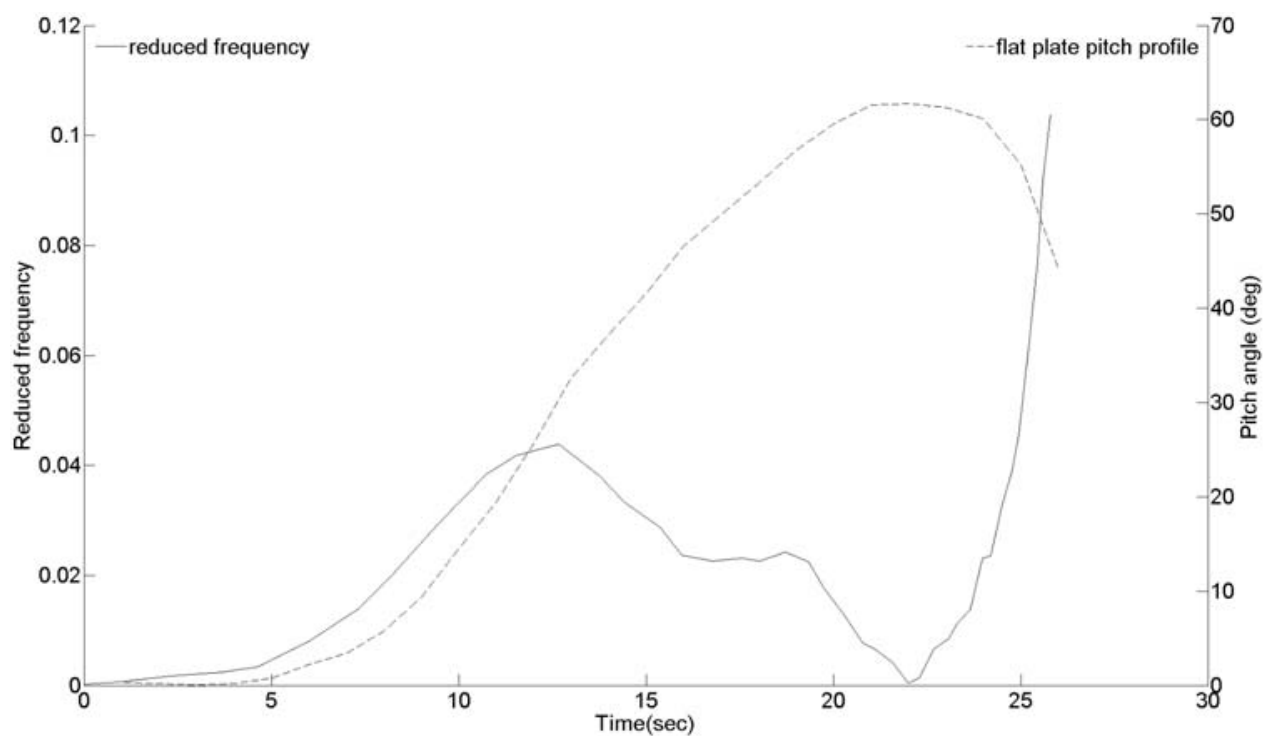

Figure 4. Flat plate pitch angle (dash line) and reduced frequency (solid line) profiles used in the present experiments. Note that absolute value of the pitch rate has been used in this plot. For time greater than 22 seconds the pitch rate is negative.

\section{B. Dye Injection Flow Visualization}

Fig. 5 shows the flow visualization results for selected phases at two span-wise locations. The top sequence was filmed with dye injected at $50 \%$ span, while the bottom sequence was filmed with dye injected at $75 \%$ span. The images at the 50\% span indicate there is no LEV, but rather a large separation bubble. The phase 13 and 16 images at the $50 \%$ span, which correspond to convective time 16 and 20, show a red streamline that came over the top of the airfoil and was vectored down, ultimately changing direction by almost $90^{\circ}$. Vortex shedding was not present during the motion, while the flow appeared to be mostly separated near phase 16 (convective time 20) in both series.

The images at the $75 \%$ span demonstrate the presence of strong tip vortices that affect a large fraction of the flat plate's span. This could explain Cory and Tedrake's ${ }^{1}$ glider's ability to perch successfully; the large separated region likely produces a higher $C_{D}$ than normal force at the end of the maneuver, which causes the glider to decelerate rapidly. The images at the $75 \%$ span illustrate the complexity of the flow field. For the present conditions of Reynolds number and reduced frequency the flow field cannot be reasonably approximated as 2D, especially near the tip of the flat plate. 

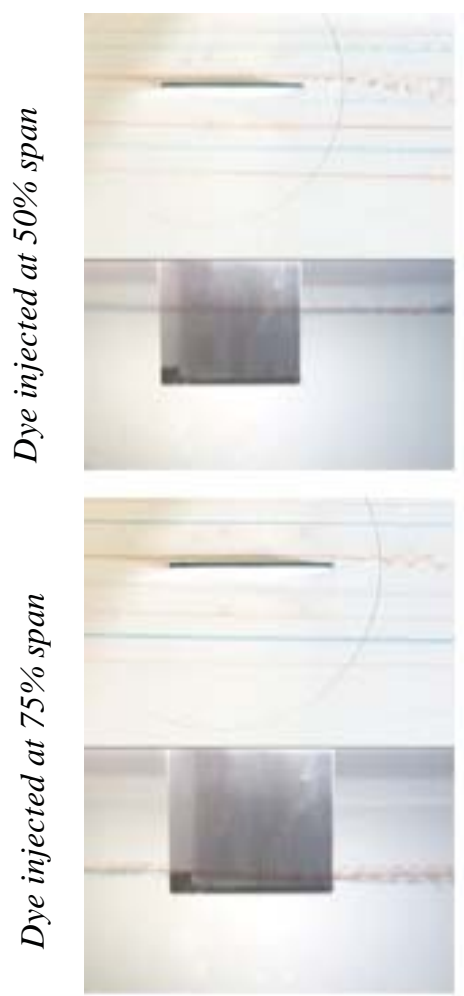

Phase 1, $\alpha=0^{\circ}, t_{c}=1$
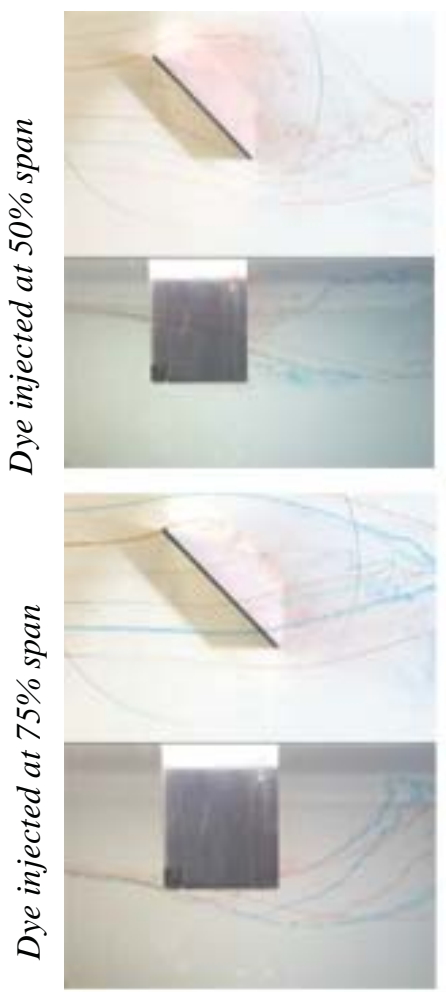

Phase 16, $\alpha=47^{\circ}, t_{c}=20$
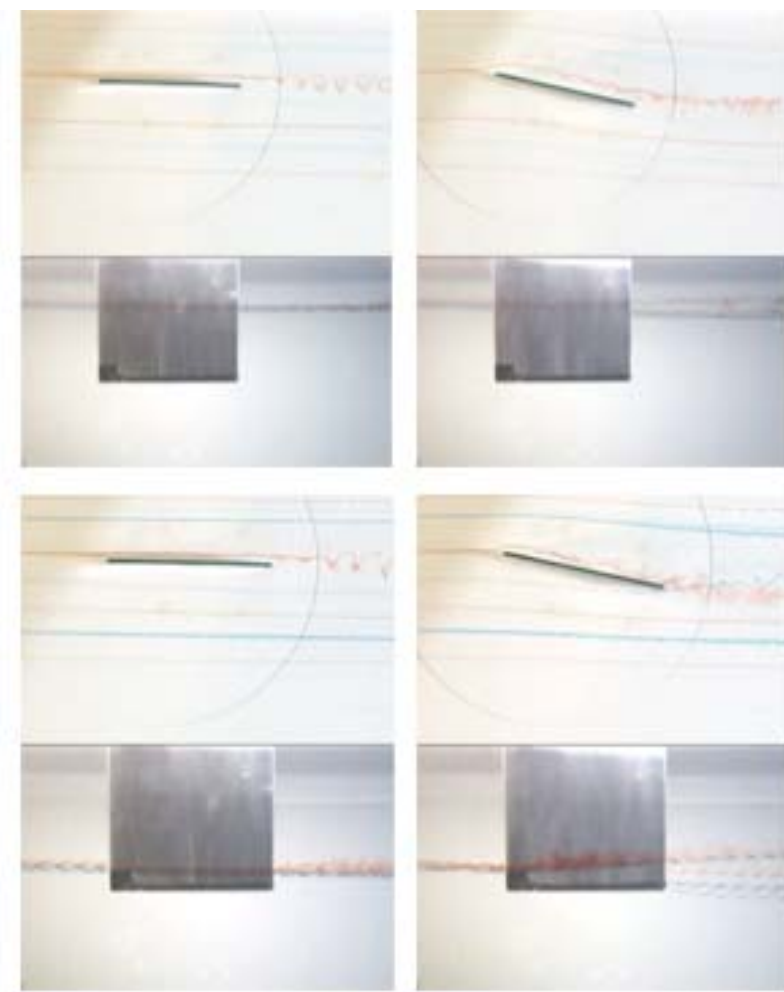

Phase 6, $\alpha=2^{\circ}, t_{c}=8$
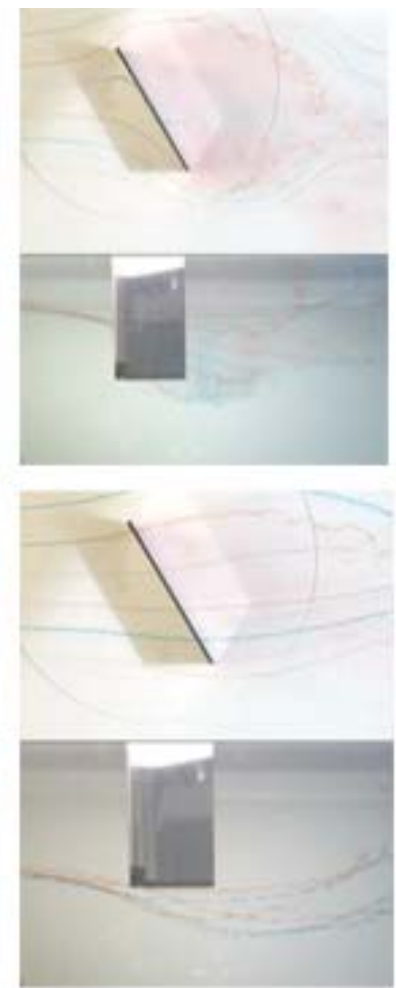

Phase 20, $\alpha=60^{\circ}, t_{c}=25$

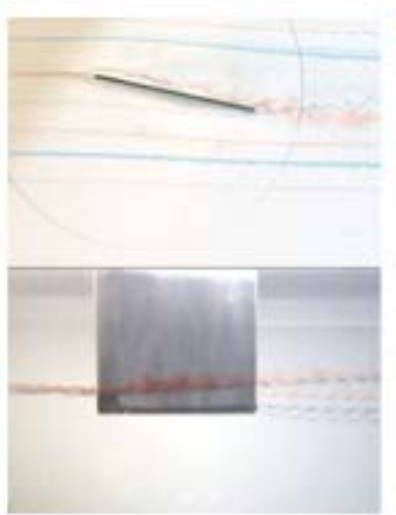

Phase 10, $\alpha=15^{\circ}, t_{c}=13$
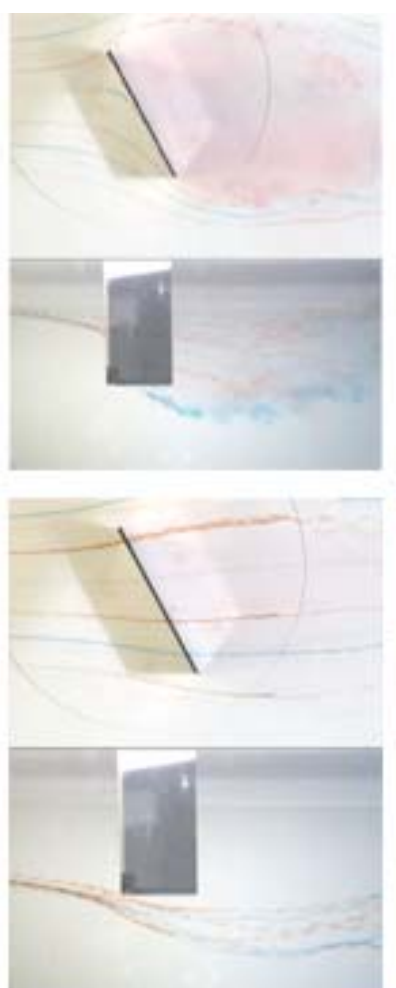

Phase 23, $\alpha=61^{\circ}, t_{c}=29$
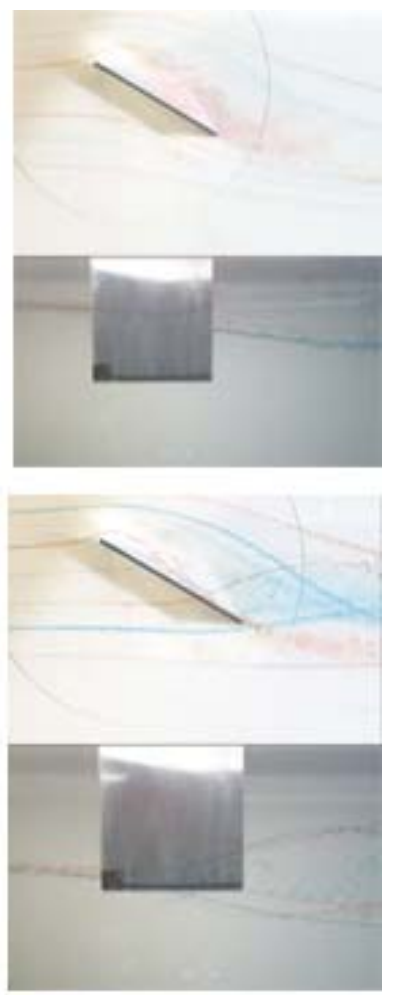

Phase 13, $\alpha=33^{\circ}, t_{c}=16$
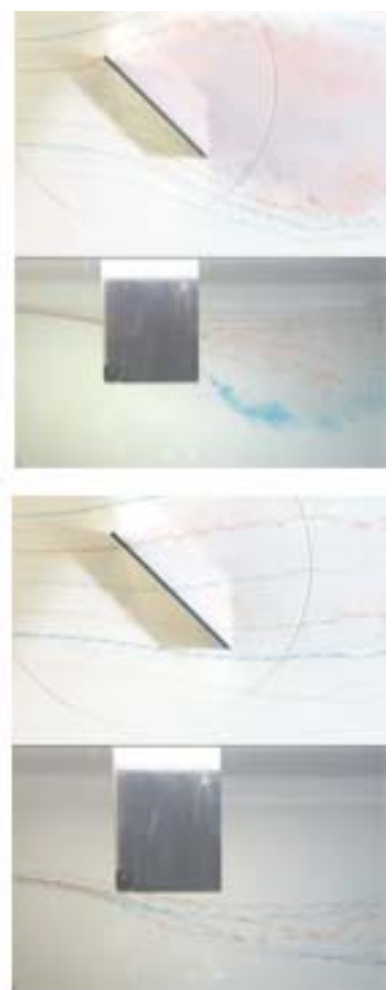

Phase 26, $\alpha=41^{\circ}, t_{c}=33$

Figure 5. Flow visualizations of the perching motion. The top image sequence was taken with dye injected at $50 \%$ span, and the bottom image sequence was taen with dye injected at $75 \%$ span. 


\section{PIV}

Fig. 6 shows contours of normalized x-velocity for the $50 \%$ and $75 \%$ spanwise locations, while Fig. 7 shows
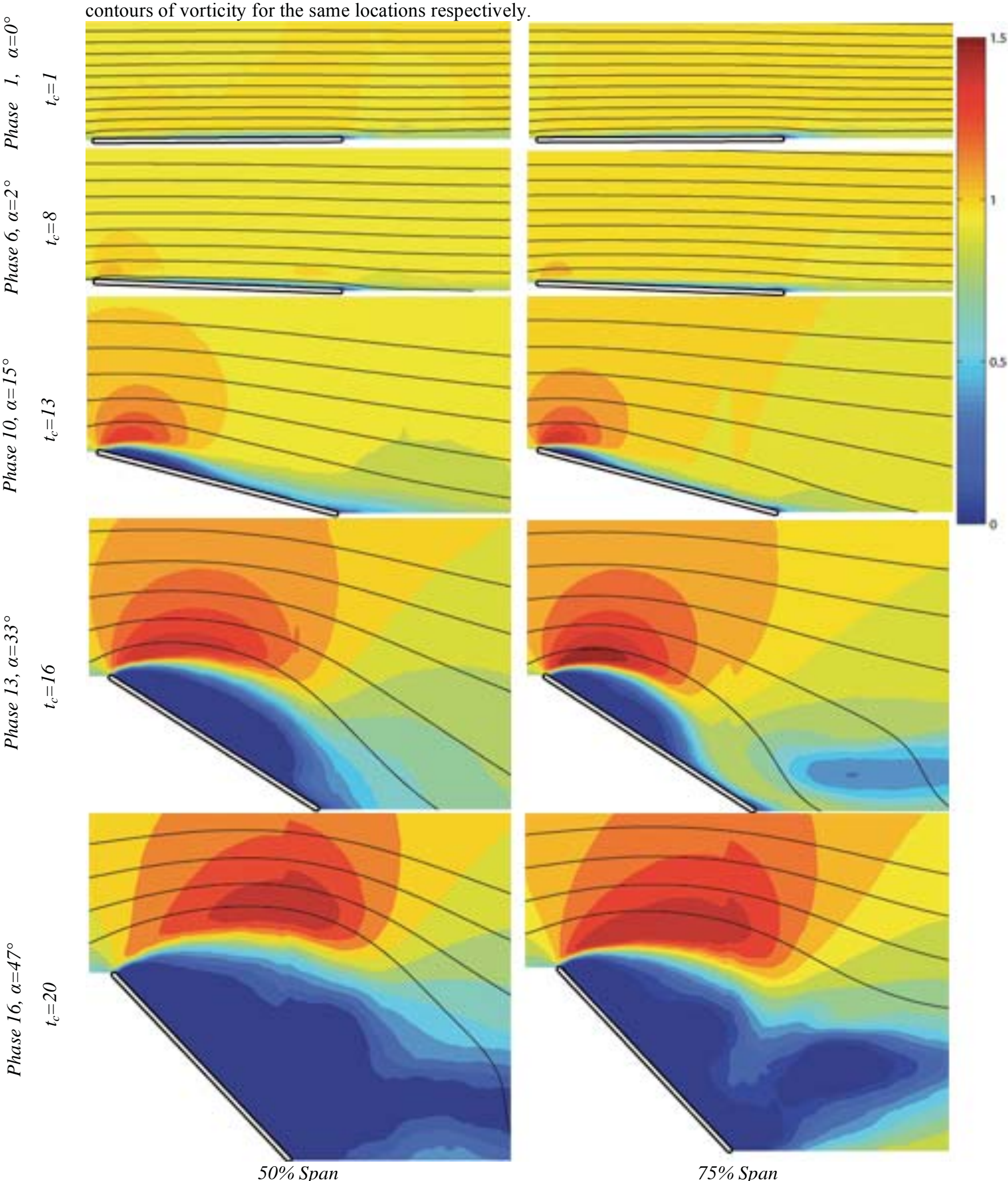

$75 \%$ Span

7

American Institute of Aeronautics and Astronautics 

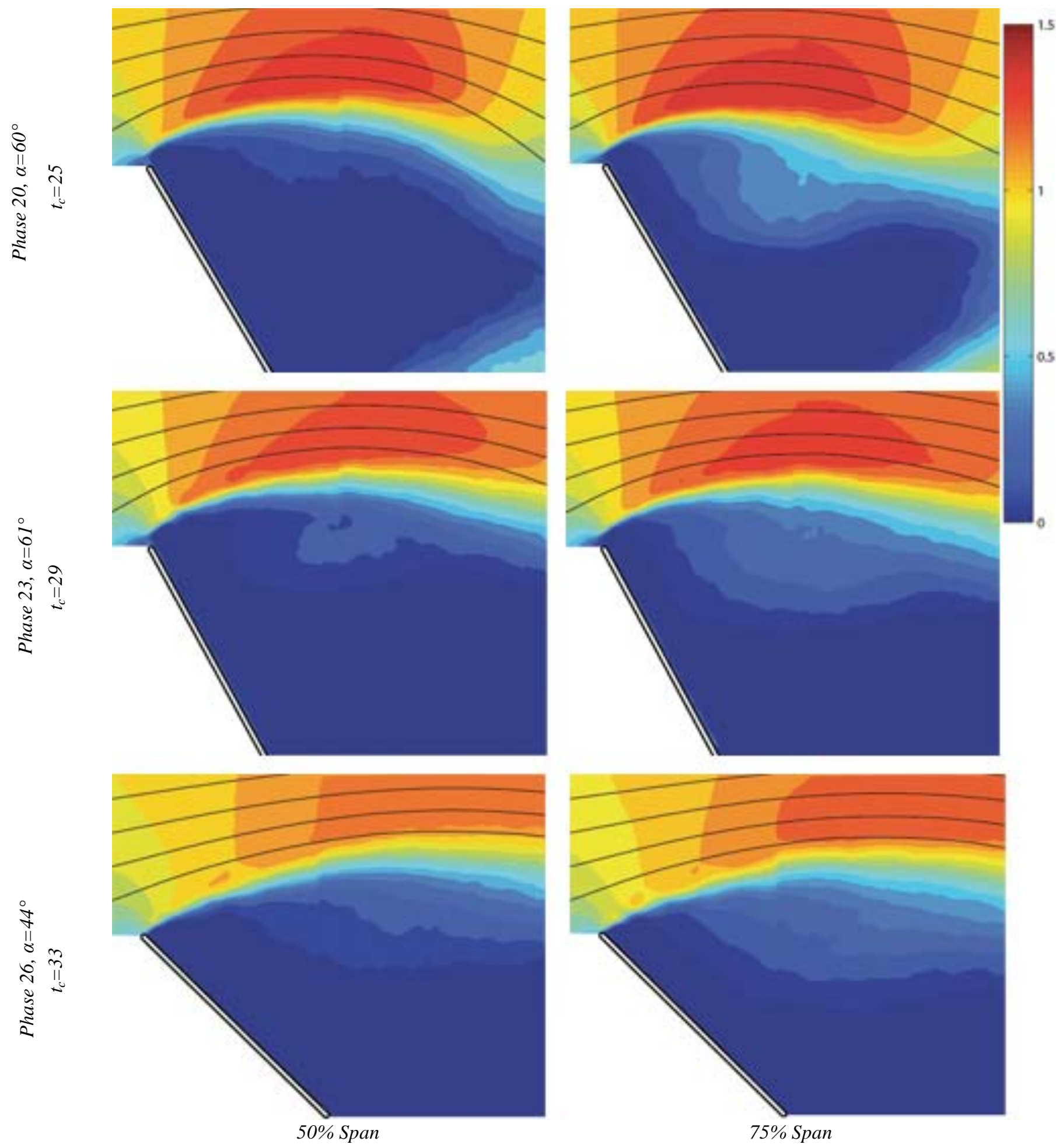

Figure 6: Contours of normalized x-velocity. 



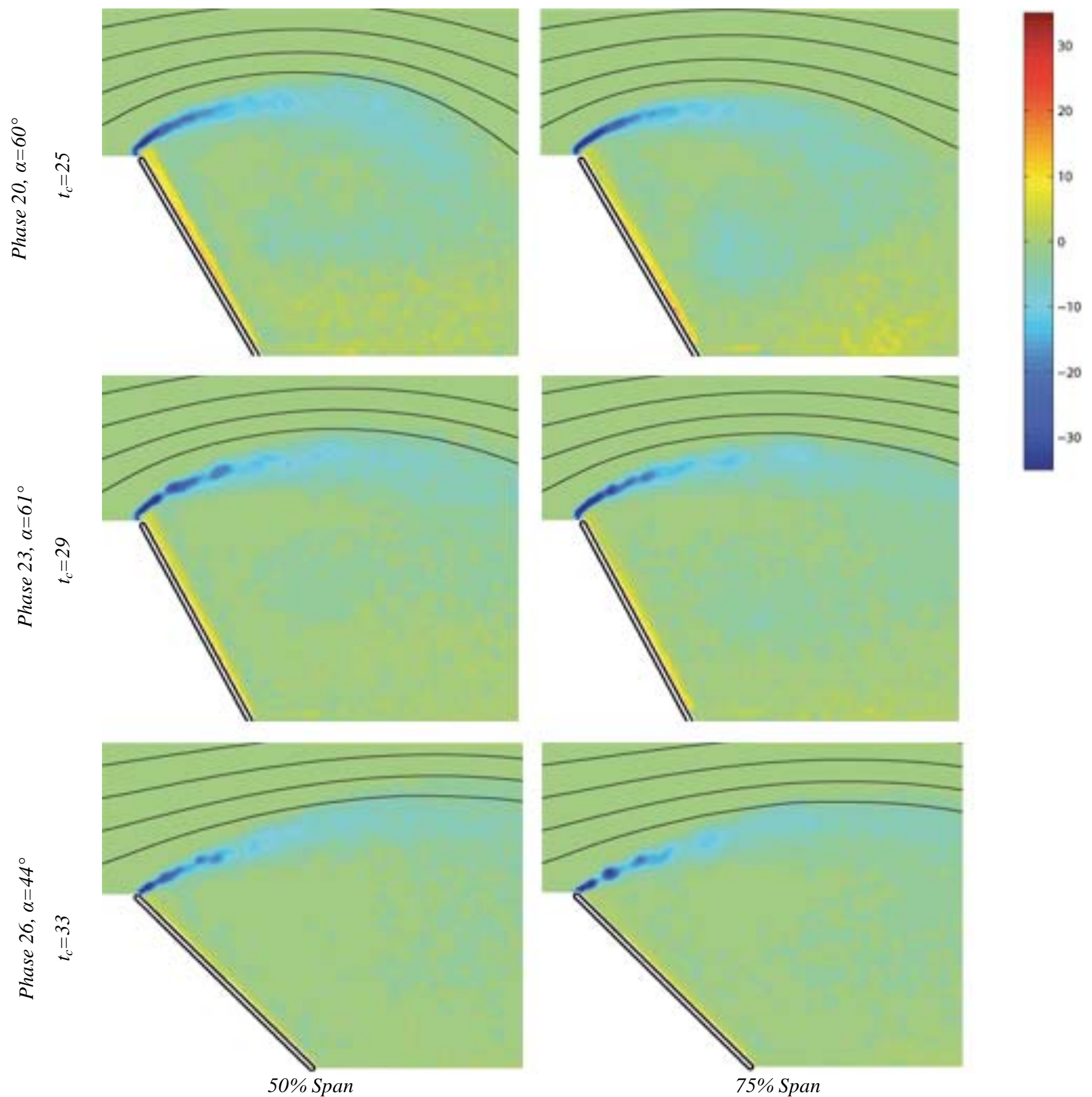

Figure 7: Contours of vorticity, [1/s].

In both figures the PIV results are for the same convective time (phase) as shown in the flow visualization results in Fig. 5. Fig. 6 illustrates the formation, growth, and detachment of the separation bubble. The differences in PIV results between the two spanwise locations are notable. The separated region grows faster at the $50 \%$ span location. There is a velocity deficit at the $75 \%$ span phase 13 and 16 images corresponding to the core of a shed TEV. This deficit is not present in the corresponding $50 \%$ span image. 


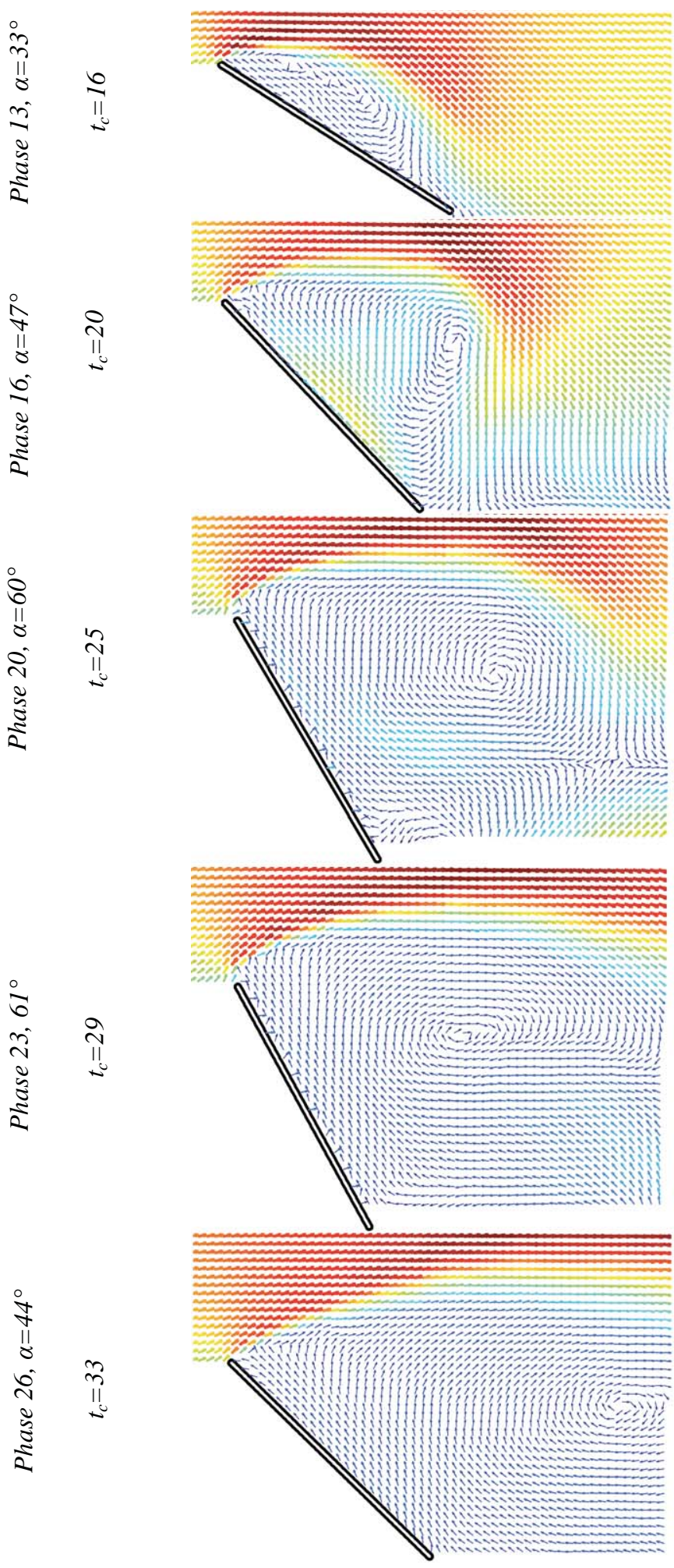

$50 \%$ Span
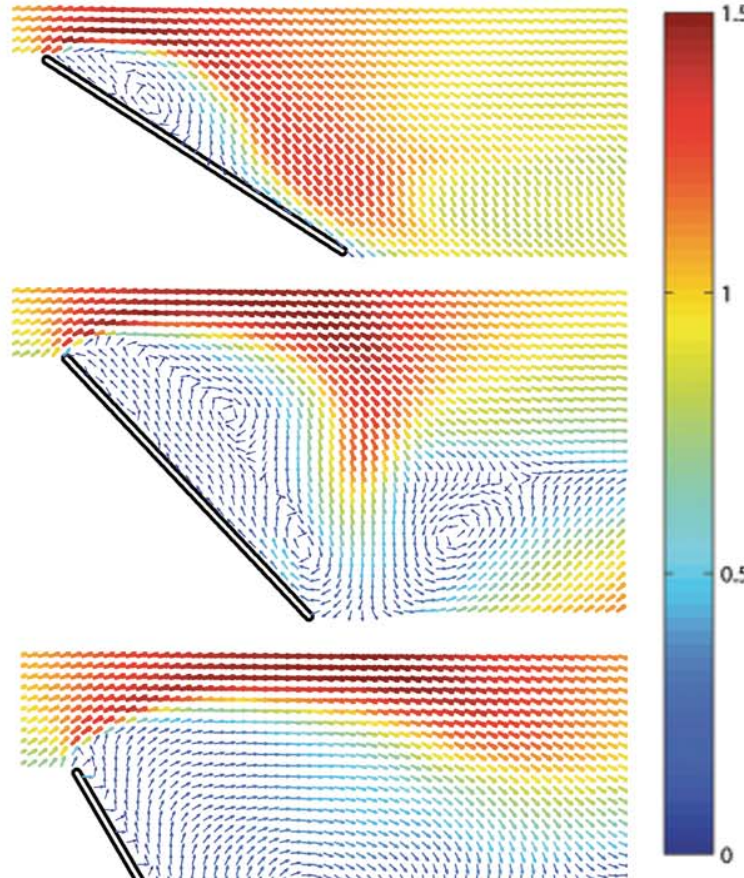

0.5
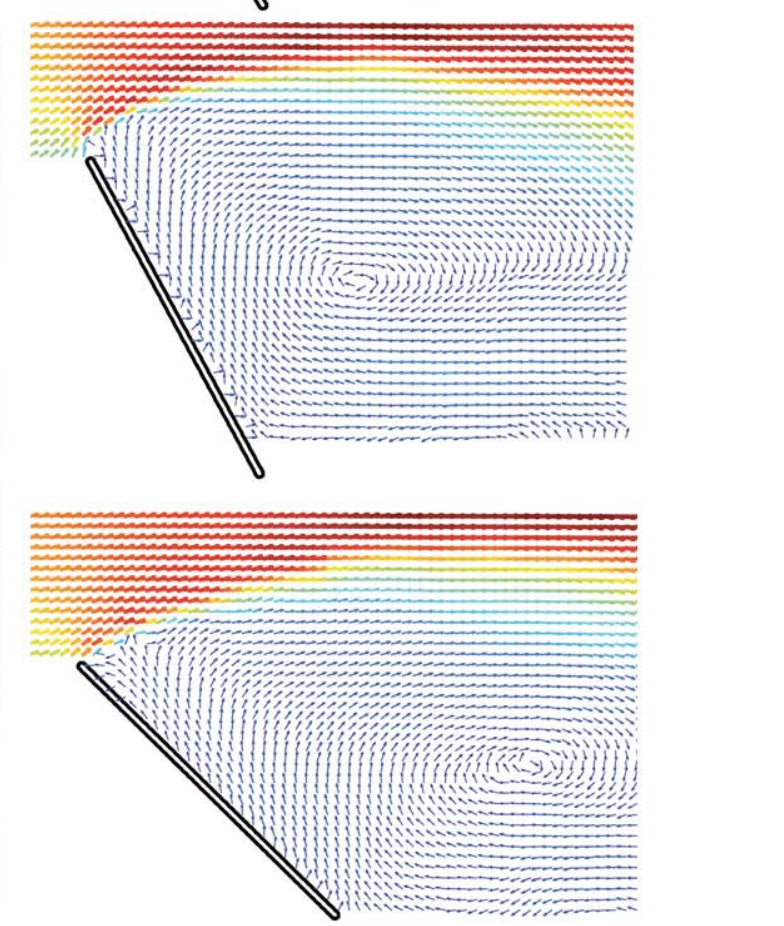

$75 \%$ Span

Figure 8: Velocity vectors colored by magnitude normalized to the freestream. Vector lengths do not correspond to flow speed at a given location. 
Fig. 7 clarifies the differences between the spanwise locations. Vortex structures are much more prevalent at the $75 \%$ span location in the phase 16 and 20 images (convective time 20 and 25 respectively). However the final flow topology at the end of the maneuver in phase 26 (convective time 33) is very similar. Span-wise location is an important factor in determining the transient flow topology.

Fig. 8 shows the velocity vectors downstream of the flat plate revealing the position of the center of the recirculation zone. It is important to note that the vector lengths are not representative of flow speed; they show only flow direction. Vector color shows the flow speed. This was done to elucidate the areas of rotation, though the angular velocity or vorticity are very small. Fig. 8 is consistent with results shown above; vertical flow features develop earlier at the $50 \%$ span location than at the $75 \%$ span location. Phase 16 (convective time 20) shows the flow is becoming fully separated at $50 \%$ span, while the flow at $75 \%$ span is more attached and a TEV is being

convected downstream. The sequences at both locations show the center of the recirculation zone being convected downstream by phase 26 (convective time 33 ).

\section{Conclusion}

This study investigated the aerodynamics of a successful perching maneuver at $R e=35,000$. The kinematics are scaled from Cory and Tedrake's ${ }^{1}$ data for use in the water channel. Two span-wise locations provide insight into the development of separation, three-dimensionality and wing tip vortex of the flow. The flow visualizations suggest a largely 3D flow for pitch angles greater than 30 degrees, with formation of a large fully 3D separated flow region. No leading edge vortex formation is observed, rather a high-shear region bounds the separated flow, and the flow speed in the recirculation region is very low. PIV and flow visualization data show that the wing tip vortex forms at a pitch angle of approximately 30 degrees and is characterized by a low velocity region at the core of the vortex.

Future work should include direct force measurements using a force sensor. These results would be compared with force measurements by Cory and Tedrake ${ }^{1}$ obtained in their successful perching experiments, and would provide valuable information about how the presence of LEVs, TEVs, and separated regions affect lift and drag. 


\section{References}

${ }^{1}$ Cory, R. and Tedrake, R., "Experiments in Fixed-Wing UAV Perching," Proceedings of the AIAA Guidance, Navigation, and Control Conference, AIAA, 2008.

${ }^{2}$ McCroskey, W. J. (1981). The phenomenon of dynamic stall. NASA TM-81264.

${ }^{3}$ Ol, M. V., "The High-Frequency, High-Amplitude Pitch Problem: Airfoils, Plates and Wings,” AIAA Paper 2009-3686.

${ }^{4}$ Eldredge, J., Wang, C., and O1, M. V., “A Computational Study of a Canonical Pitch-up, Pitch-down Wing Maneuver,” AIAA Paper 2009-3687. 39th AIAA Fluid Dynamics Conference, San Antonio, TX, June 2009.

${ }^{5}$ Wickenheiser, A., Garcia, E., Waszak, and M. O1, "Longitudinal dynamics of a perching aircraft concept," Proc. SPIE - Int. Soc. Opt. Eng. (USA), Vol. 5764, No. 1, 2005, pp. $192-202$.

${ }^{6}$ Hoburg, W., Tedrake, R., “System Identification of Post Stall Aerodynamics for UAV Perching,” AIAA InfoTech @A Aerospace Conference, Seattle, WA, 2009.

${ }^{7}$ Baik, Y. S, et al., "Experimental Investigation of Pitching and Plunging Airfoils at Reynolds Number between $1 \times 10^{4}$ and 6x104," AIAA Paper 2009-4030. 39th AIAA Fluid Dynamics Conference, San Antonio, TX, June 2009.

${ }^{8}$ Bernal, Luis., "Lab III - PIV Velocity Measurements in the Wake of a Circular Cylinder,”Aero 521.

${ }^{9}$ Ol, M.V., Cox, C., Granlund, K and Bernal, L.P., "Experiments on Abstractions of Perching," AIAA Paper 2010-4943, 40th AIAA Fluid Dynamics Conference, Chicago, IL, June 2010. 